\title{
A mesostructured hybrid CTA-silica carrier for curcumin delivery
}

\author{
Marta Gallo $\mathbb{D}^{1} \cdot$ Fabio Giudice $^{1} \cdot$ Mauro Banchero ${ }^{1} \cdot$ Silvia Ronchetti $^{1} \cdot$ Luigi Manna $^{1} \cdot$ Barbara Onida $^{1}$
}

Received: 21 May 2020 / Revised: 10 July 2020 / Accepted: 17 July 2020 / Published online: 27 July 2020

(c) The Author(s) 2020

\begin{abstract}
Curcumin is a natural active principle with antioxidant, antibacterial and anti-inflammatory properties. Its use is limited by a low water solubility and fast degradation rate, which hinder its bioavailability. To overcome this problem, curcumin can be delivered through a carrier, which protects the drug molecule and enhances its pharmacological effects. The present work proposes a simple one-pot sol-gel synthesis to obtain a hybrid carrier for curcumin delivery. The hybrid consists of a mesostructured matrix of amorphous silica, which stabilizes the carrier, and hexadecyltrimethylammonium (CTA), a surfactant where curcumin is dissolved to increase its water solubility. The carrier was characterized in terms of morphology (FESEM), physicochemical properties (XRD, FTIR, UV spectroscopy) and release capability in pseudo-physiological solutions. Results show that curcumin molecules were entrapped, for the first time, in a silica-surfactant mesostructured hybrid carrier. The hybrid carrier successfully released curcumin in artificial sweat and in a phosphate buffer saline solution, so confirming its efficacy in increasing curcumin water solubility. The proposed drug release mechanism relies on the degradation of the carrier, which involves the concurrent release of silicon. This suggests strong potentialities for topical administration applications, since curcumin is effective against many dermal diseases while silicon is beneficial to the skin.
\end{abstract}

\section{Graphical Abstract}

A simple one-pot sol-gel organic-solvent-free synthesis, at room temperature, leads to the formation of a hybrid mesostructured CTA-silica containing curcumin. When in pseudo-physiological solutions, the hybrid releases curcumin (enclosed in surfactant micelles), with enhanced solubility, and silicates. This material is a promising candidate for topical administration of drugs, since curcumin is effective against several dermatological diseases and silicon is beneficial to skin.

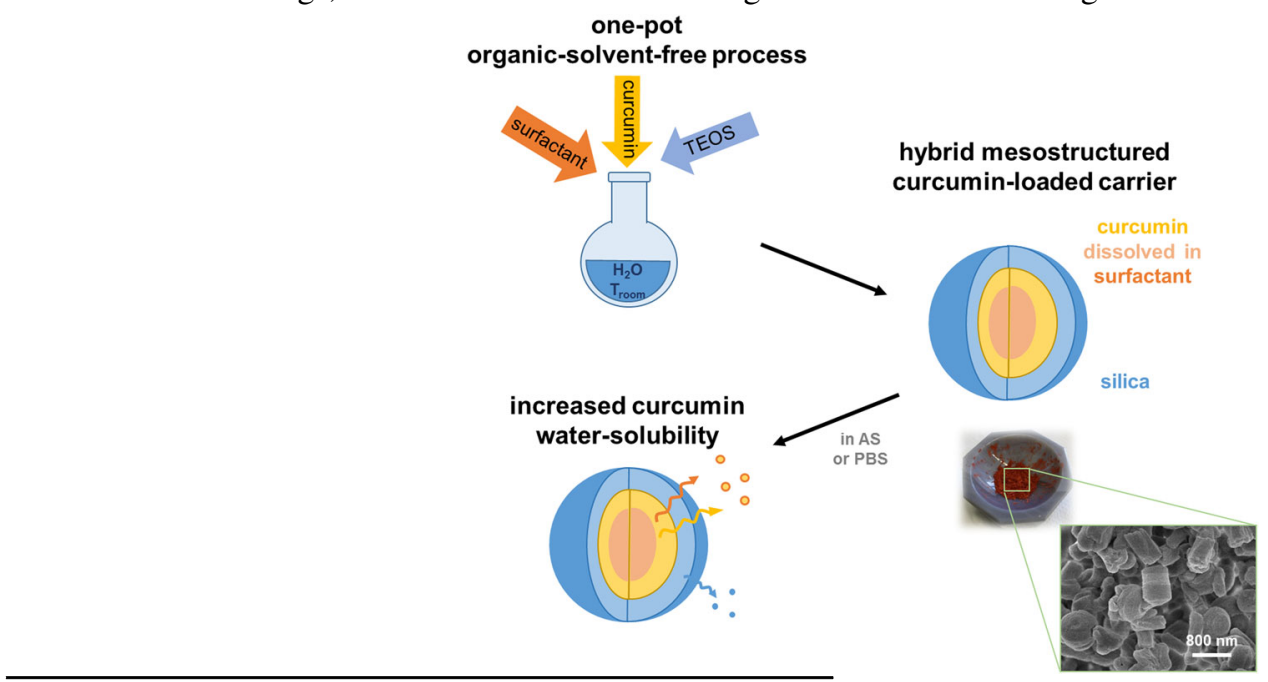

Marta Gallo

marta.gallo@polito.it

1 Dipartimento di Scienza Applicata e Tecnologia, Politecnico di Torino, Corso Duca degli Abruzzi, 24, 10129 Torino, Italy 
Keywords Drug carrier $\cdot$ Hybrid $\cdot$ Curcumin $\cdot$ Silica $\cdot$ Mesostructured $\cdot$ Topical application

\section{Highlights}

- A novel mesostructured curcumin-containing hybrid carrier was successfully synthesized.

- The hybrid enhances curcumin solubility in pseudo-physiological conditions (e.g., artificial sweat).

- Curcumin release is governed by a degradation process of the silica matrix.

- Degradation of the hybrid induces the simultaneous release of curcumin, silicates, and surfactant.

\section{Introduction}

Curcumin, a natural polyphenol derived from the turmeric rhizome, is also known as the "golden spice", because of its beneficial properties, which are widely recognized by traditional Asian medicine. However, also modern science has confirmed the effectiveness of curcumin against numerous diseases. Thanks to its functional groups (two aromatic phenolic rings and an $\alpha$ - $\beta$-diketo moiety), curcumin can act as a scavenger of reactive oxygen species and bind (covalently or not) to biomolecules [1]. This confers curcumin its unique antibacterial, anti-inflammatory, antioxidant, and wound healing properties as well as significant anticancer activity [2]. In recent years, curcumin has also been proved to be effective for skin disorders, such as psoriasis and dermatoses [3-5], and to be particularly useful to treat chronic diseases since, being it a natural molecule, it does not induce side effects even at high doses and for long-term treatments.

However, the use of curcumin is still strongly limited by its poor bioavailability, poor water solubility, and fast degradation rate (particularly in alkaline solutions and in the presence of light) [1]. To overcome these limitations, researchers have proposed to deliver curcumin by means of appropriate carriers, which are able to protect the molecule from degradation and to enhance its water solubility and bioavailability. The suggested solutions include liposomes, solid-lipid nanoparticles, polymeric nanoparticles, micelles, and mesoporous silica [5-11]. In particular, previous studies showed that micelles, which are assemblies of surfactant molecules with an inner hydrophobic core and an external hydrophilic layer, can enclose the highly hydrophobic curcumin, so protecting it from alkali degradation and increasing its water solubility and bioavailability $[6,8,12]$. Furthermore, Dhivya et al. have recently exploited the hydrophobicity of a polymer shell to incorporate curcumin in $\mathrm{ZnO}$ particles with anti-gastric cancer activity [11].

On the other hand, in recent years, nanoparticles of different materials (e.g., silica [13], metal oxide [11]) have been investigated as potential drug carriers and their properties have been extensively studied. In particular, mesoporous silica particles, with pore size ranging between 2 and $50 \mathrm{~nm}$ in diameter appear to be a valid substitute for traditional drug carriers, thanks to their versatility [13]. Indeed, mesoporous silica carriers can be loaded with curcumin, which can afterwards be released in a sustained way, so enhancing its pharmacological effects [10]. In particular, when constrained in mesopores, curcumin loses its crystallinity and turns into an amorphous form, so becoming more water soluble and, therefore, more bioavailable. The incorporation of curcumin in mesoporous silica, however, can be laborious. Indeed, curcumin can be incorporated by impregnation techniques $[10,14]$, which imply the use of harmful solvents, such as methanol or ethanol. The use of a safer alternative solvent, such as supercritical $\mathrm{CO}_{2}\left(\mathrm{scCO}_{2}\right)$, which does not present any toxicity issues, is not suitable, because curcumin has such a low solubility $\left(10^{-8}\right.$ molar fraction) in $\mathrm{scCO}_{2}$ [15], that this technique is inappropriate to achieve significant drug loading of the carrier.

Eventually, another possible approach reported in the literature consists in synthetizing silica nanoparticles directly in the presence of curcumin [16]. The obtained hybrid material, however, contains crystalline rather than amorphous curcumin and, therefore, does not exploit the potential benefits of curcumin amorphization induced by the incorporation in a silica matrix.

In order to overcome the above-cited problems, a mesostructured hybrid carrier is here proposed, which joins the advantages of micelles (drug protection and increase of water solubility) with those of amorphous silica (higher stability and possible release of silicon as orthosilicic acid). The same objective has also been attempted by other research groups [17, 18]. Kerkhofs et al. for example, synthesized silica capsules that contained micelles of P123, a surfactant able to enclose the anti-inflammatory drug flurbiprofen [17]. Also Chin and coworkers [18] synthesized a hybrid material containing hexadecyltrimethylammonium bromide (CTAB) as a surfactant, curcumin, and iron oxide nanoparticles for a targeted drug delivery. However, this last synthesis was carried out in an acidic environment, required a long time (i.e., days) and involved the use of formamide, a toxic reagent [19]. In the present work, a similar approach has been followed, using CTAB as a surfactant, but different synthesis conditions (namely alkaline) and no harmful organic solvents were employed to enclose curcumin as a model drug. To the 
knowledge of the authors, this is the first time that curcumin is incorporated in such a hybrid carrier. Interestingly, such a carrier could be exploited also for the incorporation of other hydrophobic drugs, different from curcumin.

With the aim of following a bottom-up approach and avoiding curcumin degradation, a mild, fast, and simple sol-gel synthesis was selected, which operated at room temperature, and in an organic-solvent-free aqueous medium. The carrier was prepared by adapting previous literature synthesis processes $[20,21]$, which led to the production of a hybrid mesostructured silica, in which the mesopores still contained the surfactant and curcumin. Two materials were synthesized: a reference hybrid mesostructured silica without curcumin and another one containing curcumin. The so-obtained materials were fully characterized in terms of morphology (field emission scanning electron microscopy analyses, FESEM) and of physicochemical properties (X-ray diffraction, XRD, Fourier infrared, FTIR, and UV-Visible spectroscopy on the solid). In addition, preliminary release tests in fluids simulating different physiological conditions, such as skin surface, gastric, and plasma environment, were performed.

\section{Materials and methods}

\subsection{Synthesis}

Mesostructured silica was obtained through a synthesis process inspired by previous works reported in the literature $[20,21]$. All reagents were purchased from Sigma-Aldrich (Milano, Italy) and used as received. Briefly, $0.40 \mathrm{~g}$ of hexadecyltrimethylammonium bromide (CTAB) were added under constant stirring (150 rpm) to a $0.05 \mathrm{M}$ solution of $\mathrm{NaOH}(0.19 \mathrm{~g}$ of $\mathrm{NaOH}$ in $95 \mathrm{~mL}$ of distilled water). After 40-50 min, $31.7 \mathrm{mg}$ of curcumin (Cur) were added and solubilized for $5 \mathrm{~min}$. Afterwards, $2 \mathrm{~mL}$ of tetraethyl orthosilicate (TEOS) were added dropwise. The solution, which turned into a colored slurry, was left under stirring for $3 \mathrm{~h}$. Then the precipitate was filtered from the solution, washed with distilled water, and let dry in air inside an oven at $40{ }^{\circ} \mathrm{C}$ overnight until constant weight. When the curcumin-free reference material was prepared, the procedure was the same except that no curcumin was added. The final molar ratios for the reference and the curcumin-loaded materials were, respectively: 1 TEOS:0.122 CTAB:0.536 $\mathrm{NaOH}: 589 \quad \mathrm{H}_{2} \mathrm{O}$ and 1 TEOS:0.122 CTAB:0.536 $\mathrm{NaOH}: 0.02$ Cur:589 $\mathrm{H}_{2} \mathrm{O}$.

\subsection{Characterization}

Once ground, the previously obtained powders underwent XRD analyses in a Panalytical X'Pert PRO $(\mathrm{Cu} \mathrm{K} \alpha$ radiation, Malvern Panalytical, Almelo, The Neatherlands). Data collection has been performed at $40 \mathrm{kV}$ and $40 \mathrm{~mA}$, with a solid-state detector (PIXcel1D) at small and high angles $\left(2 \theta=0.7^{\circ}-10^{\circ}\right.$ and $2 \theta=5^{\circ}-60^{\circ}$ respectively $)$. Morphology of Pt-metallized specimens was observed with a FESEM Zeiss Merlin (Oxford Instruments, Abingdon-onThames, UK). FTIR spectra were recorded at a resolution of $2 \mathrm{~cm}^{-1}$ on pelletized powders (with the addition of $\mathrm{KBr}$ ) with an Equinox 55 spectrometer (Bruker, Billerica, MA, USA) after outgassing the sample at room temperature (residual pressure of $0.1 \mathrm{~Pa}$ ). UV-Vis spectroscopy analyses on powders were performed with a Varian Cary 5000 spectrophotomer (Agilent, Santa Clara, CA, USA) equipped with a diffuse reflectance accessory. Data are reported as Kubelka-Munk function.

\subsection{Release tests}

Release tests were performed in three different solutions. A Phosphate Buffer Saline solution (PBS) $0.01 \mathrm{M}, \mathrm{pH}=$ 7.4, was obtained by dissolving commercial tablets (Sigma-Aldrich, Milano, Italy) in distilled water to mimic plasma. A $0.1 \mathrm{M} \mathrm{HCl}$ solution $(\mathrm{pH}=0.8)$, which reproduced the gastric conditions, was prepared with $\mathrm{HCl} 37$ wt.\% (Sigma-Aldrich, Milano, Italy) and distilled water. Artificial sweat (AS), which reproduced the topical environment, was prepared according to Shimamura et al. [22] by dissolving $2.34 \mathrm{~g} \mathrm{NaCl}, 0.13 \mathrm{~g} \mathrm{CaCl}_{2}, 0.10 \mathrm{~g}$ $\mathrm{MgSO}_{4}$, and $0.82 \mathrm{~g} \mathrm{KH}_{2} \mathrm{PO}_{4}$ in $800 \mathrm{~g}$ of distilled water and finally adjusting the $\mathrm{pH}$ to the value of 5.4 by adding a $0.05 \mathrm{M} \mathrm{NaOH}$ solution (all reagents were purchased by Sigma-Aldrich, Milano, Italy, except for $\mathrm{CaCl}_{2}$ that was provided by Merck, Milano, Italy). Release tests were carried out in an Erweka DT tester (Erweka, Langen, Germany) at $37^{\circ} \mathrm{C}$ under stirring (100 rpm). Absorption of the release medium was constantly monitored at $425 \mathrm{~nm}$ for PBS, $429 \mathrm{~nm}$ for the $\mathrm{HCl}$ solution, and $427 \mathrm{~nm}$ for AS for $20 \mathrm{~h}$ in a Lambda 25 spectrophotometer (Perkin Elmer, Waltham, MA, USA).

\section{Results}

\subsection{Synthesis}

The synthesis process led to the production of $\sim 650 \mathrm{mg}$ of the reference material; a similar amount was obtained as far as the curcumin-loaded material is concerned. As shown in Fig. 1, the reference material (hereafter named "Meso_SiO 2 ") appeared as a white powder, while the one containing curcumin (named "Meso_SiO ${ }_{2}$ Cur") displayed a red color, which visually confirms the presence of curcumin inside the material. It is worth noting that crystalline curcumin is not 
Fig. 1 Photos of Meso_SiO Meso_SiO 2 Cur (b), and crystalline curcumin $(\mathbf{c})$ powders

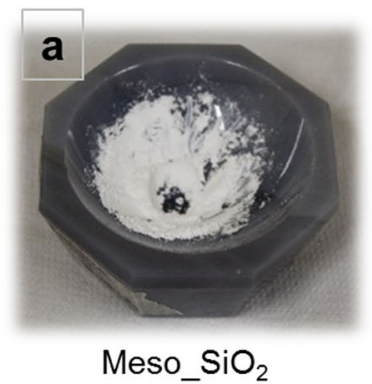

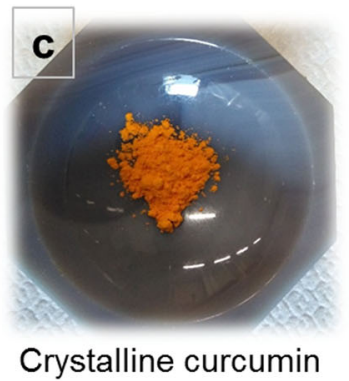
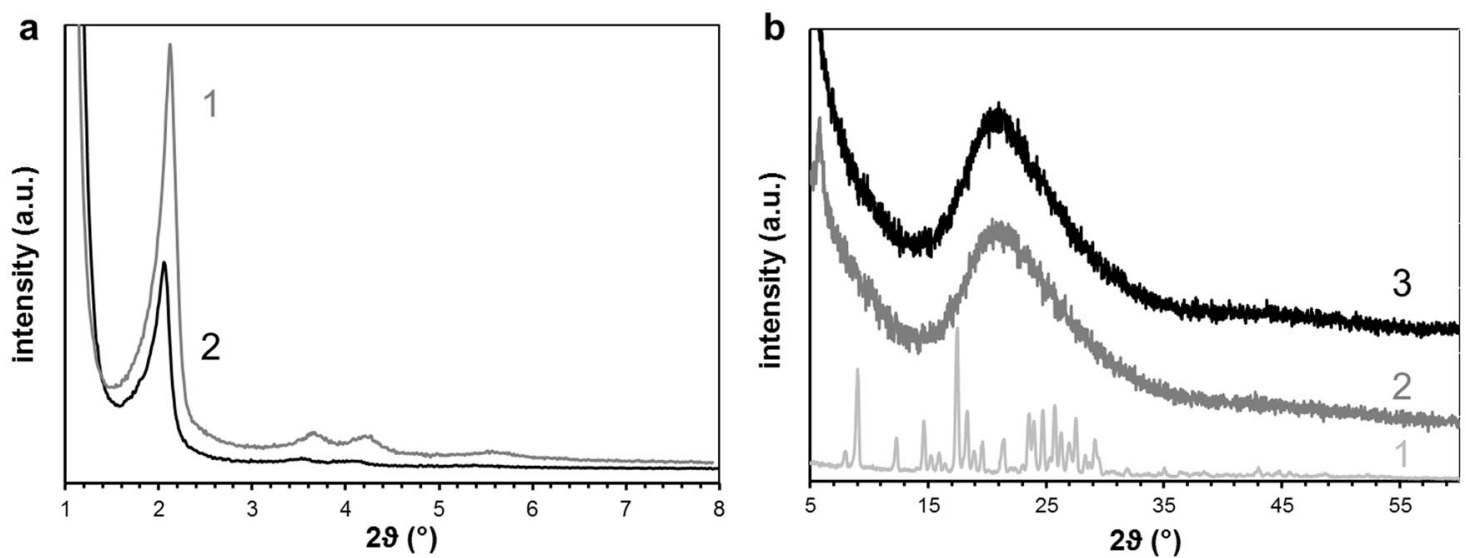

Fig. 2 a XRD patterns of Meso_SiO ${ }_{2}(1)$ and $\mathrm{Meso}_{-} \mathrm{SiO}_{2}$ CCur (2) at small angles. b XRD patterns of crystalline curcumin (1), Meso_SiO ${ }_{2}(2)$, and Meso_SiO 2 Cur (3) at high angles

red, but rather bright yellow-orange (Fig. 1c). The darker color of Meso_SiO ${ }_{2}$ Cur was ascribed to the deprotonation of curcumin, which typically causes this bathochromic effect [1]. In fact, since the synthesis takes place in a basic solution, curcumin in the final material is in its deprotonated form.

Moreover, it must be underlined that the filtered synthesis solution was reddish (Appendix Fig. 10a), which suggests that part of the curcumin was not incorporated in the material, but remained in the synthesis solution.

Finally, it is worth noting that the present synthesis procedure differs from similar works reported in the literature, such as the one by Chin et al. [18] since it was carried out in basic conditions (instead of the acidic ones), required a shorter time (few hours rather than days) and did not imply the use of toxic organic reagents, such as formamide.

\subsection{Characterization}

Figure 2a reports the XRD patterns obtained at small angles for the reference material Meso__SiO ${ }_{2}$ and for the one containing curcumin, Meso_SiO ${ }_{2}$ Cur. In both spectra, three peaks are visible, respectively at $2 \theta$ values of $2.13,3.64$, and 4.27 for Meso_SiO 2 and of 2.07, 3.54, and 4.08 for Meso_SiO ${ }_{2}$ Cur. For both samples, the three peaks evidence the $2 \mathrm{D}$ hexagonal symmetry of a mesostructured material and they correspond to the reflections (100), (110), and (200), respectively. In addition, a fourth peak is visible in the pattern of Meso_SiO ${ }_{2}$ at $2 \theta$ equal to 5.61 , which is due to the reflection (210).

It can be noticed that all peaks in the $\mathrm{Meso}_{-} \mathrm{SiO}_{2} \mathrm{Cur}$ sample are shifted toward lower angles with respect to those of Meso_SiO ${ }_{2}$. As a consequence, the cell parameter $a$, which can be calculated from the knowledge of $d_{(100)}$ and on the basis of the $a=2 d_{(100)} / \sqrt{ } 3$ geometrical assumption [23], is equal to $4.80 \mathrm{~nm}$ for Meso_ $\mathrm{SiO}_{2}$ and $4.95 \mathrm{~nm}$ for Meso_SiO ${ }_{2}$ Cur, respectively.

Figure $2 \mathrm{~b}$ shows the diffraction pattern at high angles of the two synthesized materials and of the crystalline curcu$\mathrm{min}$ as received. While crystalline curcumin displays several peaks, where the major ones are at $2 \theta=17.51$ and $2 \theta$ $=9.03$, no peaks are observed either in the reference material or in the curcumin-loaded one.

Figure 3 displays the FESEM images of the reference material (Fig. 3a) and of the curcumin-loaded one (Fig. 3b). The particles of Meso_ $\mathrm{SiO}_{2}$ are characterized by a regular morphology, with hexagonal shape and a size of about 
Fig. 3 FESEM images of Meso_SiO $\mathrm{Si}_{2}$ (a) and Meso_SiO 2 Cur (b)
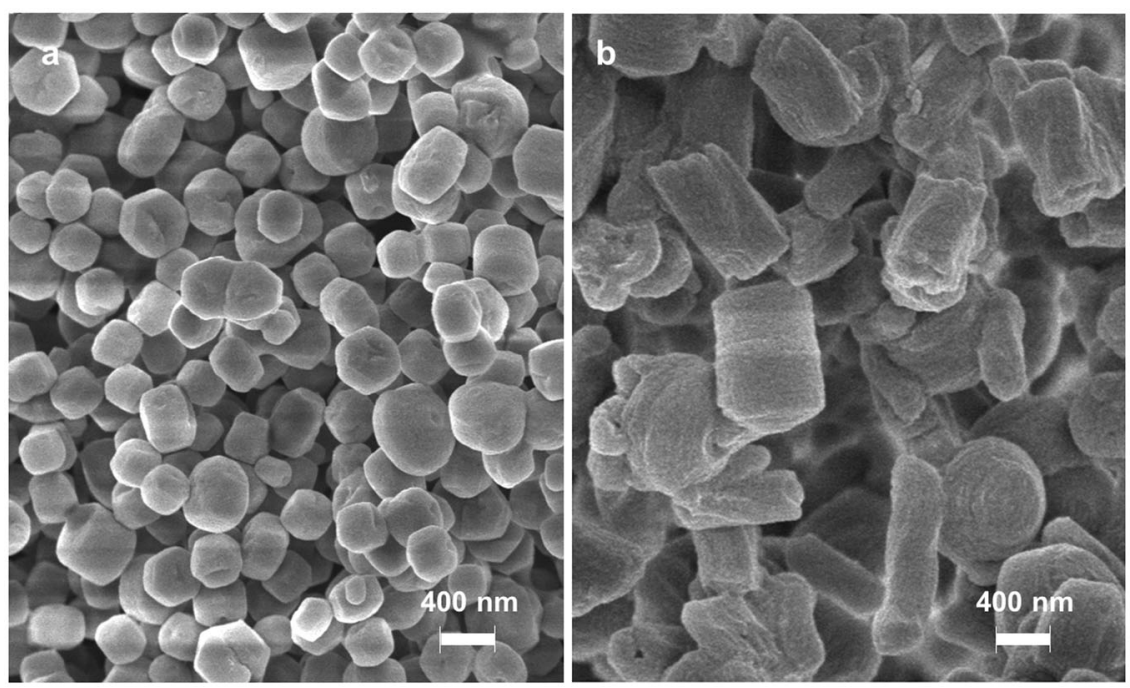

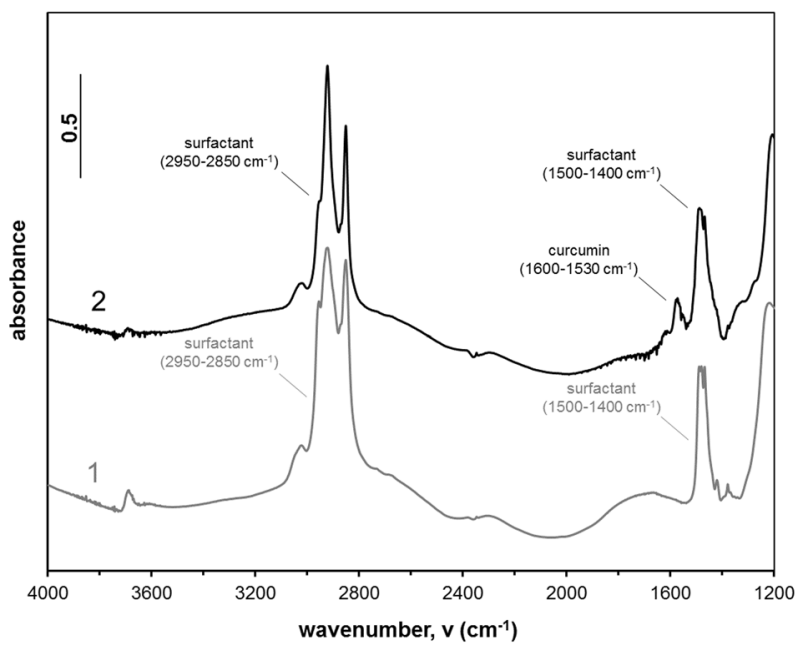

Fig. 4 FT-IR spectra of Meso_SiO ${ }_{2}$ (dark gray, 1) and $\mathrm{Meso}_{-} \mathrm{SiO}_{2} \_\mathrm{Cur}$ (black, 2) outgassed at room temperature

500-600 nm. Meso_SiO 2 Cur, instead, shows rod-like particles long up to $700-800 \mathrm{~nm}$ (some of them with a hexagonal-shaped section) as well as some discoid particles of $500-600 \mathrm{~nm}$ in diameter.

FTIR spectra of the synthesized materials, which were outgassed at room temperature, are reported in Fig. 4. In the spectrum of Meso_SiO ${ }_{2}$, the weak peak at about $3700 \mathrm{~cm}^{-1}$ is due to the stretching mode of silanols, while bands in the range $2950-2850 \mathrm{~cm}^{-1}$ and $1500-1400 \mathrm{~cm}^{-1}$ are attributed, respectively, to the stretching modes of the $-\mathrm{CH}_{2}-$ and $-\mathrm{CH}_{3}$ groups and to the bending modes of the $\mathrm{N}-\mathrm{CH}_{2}-$ groups of the surfactant molecules [24]. The spectrum of Meso_SiO ${ }_{2}$ Cur is characterized by the same bands as the reference material as well as other additional peaks, which are observed in the range between 1600 and $1530 \mathrm{~cm}^{-1}$ and

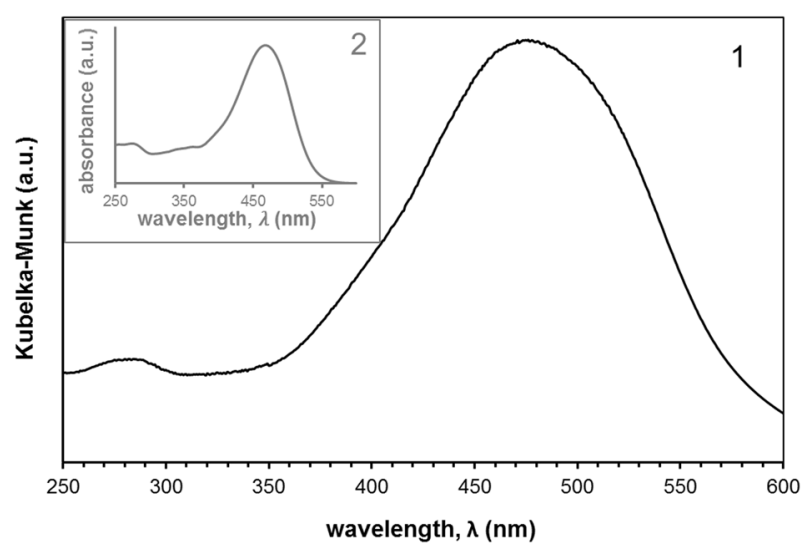

Fig. 5 UV-Vis spectra of Meso_SiO 2 Cur powder (1). Inset (2) shows the UV-Vis spectrum of curcumin $(7 \mathrm{ppm})$ in a $0.05 \mathrm{M}$ solution of $\mathrm{NaOH}$

are ascribed to the stretching vibrations of the aromatic rings of curcumin $[25,26]$.

Figure 5 reports the UV-Vis spectrum of the Meso_$\mathrm{SiO}_{2}$ Cur powder (curve 1); since $\mathrm{Meso} \_\mathrm{SiO}_{2}$ did not present any peak in the analysed range, its spectrum is not reported for sake of simplicity. The spectrum reported in Fig. 5 reveals a broad band centred at $476 \mathrm{~nm}$ (coherently with the red color of the powder). The inset in Fig. 5 displays the adsorption spectrum of curcumin dissolved in a $0.05 \mathrm{M} \mathrm{NaOH}$ alkaline solution (with the addition of around $10 \mathrm{wt} . \%$ ethanol in order to increase the solubility of curcumin at a detectable level), for comparison. The spectra of the solid powder and the curcumin alkaline solution are quite similar. The band of the curcumin basic solution $(468 \mathrm{~nm})$ is shifted only by a few nanometers with respect to that of the solid powder $(476 \mathrm{~nm})$. 


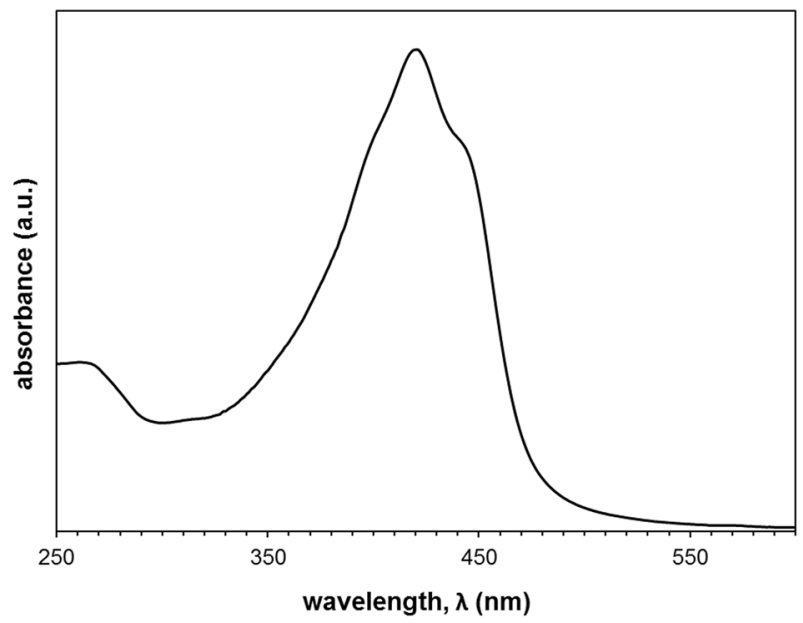

Fig. 6 UV-Vis absorption spectra of artificial sweat solution (AS) at the end of the release test from Meso_SiO ${ }_{2}$ Cur

\subsection{Release tests}

A first result is that no residual material was observed at the end of the tests in PBS and AS, while a yellow residual powder was retrieved after the test in $\mathrm{HCl}$ solution.

Figure 6 reports the absorption spectrum of the AS solution, which was measured at the end of the release test, i.e., after immersing $\mathrm{Meso}_{-} \mathrm{SiO}_{2}$ C Cur at $37^{\circ} \mathrm{C}$ for $20 \mathrm{~h}$. A similar spectrum was obtained at the end of the release test in PSB. A structured absorption with a maximum at $422 \mathrm{~nm}$ is observed, due to the dissolved curcumin that was released from the material upon immersion.

As far as the kinetics of release is concerned, results are reported in Fig. 7: the amount of the curcumin released from the carrier in different media is represented as a function of time.

In the $\mathrm{HCl}$ solution, negligible release was observed. On the opposite, curcumin was released from the carrier both in PBS and in AS. In detail, in PBS the release started $1 \mathrm{~h}$ after the immersion of the sample, then the amount of released curcumin rapidly increased until a plateau was reached after $5 \mathrm{~h}$. When the carrier was immersed in AS, the curcumin was released after a $2 \mathrm{~h}$ delay. Again, curcumin was then rapidly released, and the plateau was reached at around $12 \mathrm{~h}$. The total amount of released curcumin can be used to evaluate the content of curcumin in the carrier, which corresponds to about 5 wt. $\%$.

As a comparison, the dissolution of crystalline curcumin in PBS is also reported in Fig. 7: no release of the drug was detected for the whole duration of the test. Similar results (not reported) were obtained for the dissolution of crystalline curcumin in the other media (AS and $\mathrm{HCl}$ solution).

\section{Discussion}

\subsection{Characterization}

Both synthesized materials are amorphous with an ordered hexagonal mesostructure typical of MCM-41 silica [21], as revealed by the peaks at small angles in the X-ray diffraction patterns (Fig. 2a). The corresponding cell parameters, derived from the position of the peak $d_{(100)}$, show a slightly higher value for Meso_SiO 2 CCur $(4.95 \mathrm{~nm})$ with respect to Meso_SiO ${ }_{2}(4.80 \mathrm{~nm})$, so suggesting that the presence of curcumin causes a swelling of the micelles. It is worth noting that the curcumin present in the hybrid carrier is not crystalline (Fig. 2b), contrary to what observed for curcumin in silica nanoparticles [16], and, therefore, it is expected to be more bioavailable.

The presence of curcumin also causes the change of the particles morphology observed by FESEM (Fig. 3). Whereas the hexagonal shape typical of MCM-41 silica is partially maintained in the reference material, the particles become more elongated or assume a discoid-shape when curcumin is embedded. This is coherent with what observed by other authors when a dye was added during the synthesis of mesoporous silica [27]. The above-mentioned impact on the particle shape that results from drug incorporation is a proof that curcumin has an influence on the co-assembly process and may act as a co-templating agent.

FT-IR results confirm the presence of both surfactant and curcumin (Fig. 4). The weak intensity of the band due to silanols is in agreement with the expected interface between silica and micelles for materials synthesized in alkaline conditions, i.e., a strong interaction between the negatively charged silica network and the positively charged surfactant.

In a similar manner the keto-enol and the hydroxyl groups of curcumin are deprotonated (therefore they are negatively charged) and can interact with the positivecharged surfactant. This means that the interactions of curcumin inside the carrier could be not only hydrophobic, but also ionic. This implies that, probably, curcumin is not segregated in the core of the surfactant micelles, but it is rather located more closely to the interface between the surfactant and silica, similarly to what observed in a previous work for another dye [20]. Furthermore, this could explain why the embedding of curcumin does not cause only a simple swelling of the hybrid mesostructure (as observed by the XRD analyses in Fig. 2a), but also alters the morphology of the final particles (FESEM images in Fig. 3b), so acting as a co-templating agent.

Another evidence of the strong interactions involving curcumin inside the carrier is provided by washing the Meso_SiO 2 _Cur powder with different solvents (Appendix Figs 10,11). When washed with water, the powder did not 
Fig. 7 Curcumin release curves from Meso_SiO ${ }_{2}$ Cur in $\mathrm{HCl}$ solution (light gray, 2), PBS (dark gray, 3 ), and artificial sweat (AS, black, 4). Release curve of crystalline curcumin in PBS (dark gray bolt line, 1) is reported for comparison. Error bars refer to instrument uncertainty

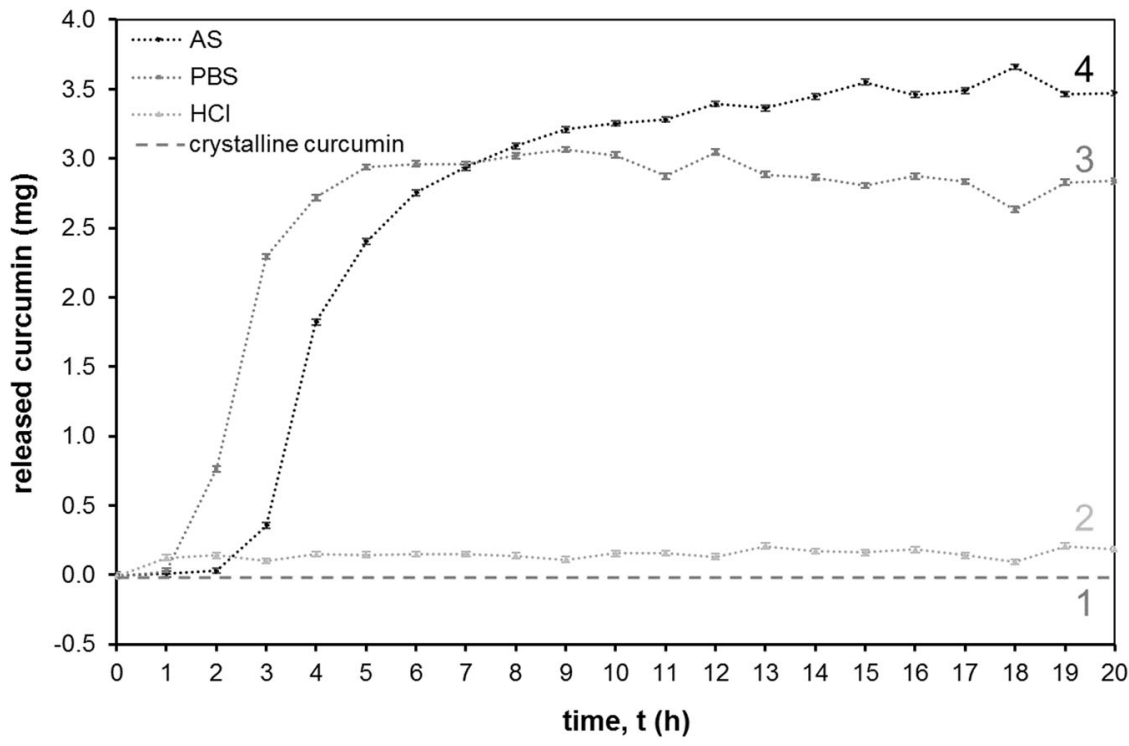

release any curcumin (Appendix Fig. 10b-c- $b^{\prime}-c^{\prime}$ ), whereas, when washed with ethanol, the leaching of curcumin was observed (Appendix Fig. 11a). These results confirm that the powder can be washed with water to remove any possible excess of synthesis reactant without removing curcumin. Since curcumin is part of the internal micelles, water does not cause any leaching. To remove curcumin from the carrier the use of a solvent with higher affinity, such as ethanol, is necessary. Interestingly, when washed with ethanol, the powder not only became white (because of the curcumin loss, Appendix Fig. 11b) but also lost its mesostructure (Appendix Fig. 12). This could be explained by supposing that ethanol is able to remove both the curcumin and partially the surfactant so inducing a loss of order in the material.

The UV-Vis spectra of the Meso_SiO ${ }_{2}$ Cur powder confirm the state of curcumin in the hybrid material. The absorption peak at around $476 \mathrm{~nm}$ (Fig. 5) is attributed to the curcumin in the deprotonated form [1]. This is coherent with the alkaline environment of the synthesis conditions and suggests that, being curcumin negatively charged, it can interact with the cationic surfactant through electrostatic interactions.

As a conclusion, it can be stated that a hybrid mesostructured material containing curcumin molecules has been successfully synthesized. According to the evidences provided by different characterization techniques, a schematic representation of the hybrid material can be sketched, as reported in Fig. 8. It can be hypothesized that curcumin does not segregate in the inner core of the hybrid material, but is rather dissolved in the surfactant and it may also be located at or close to the interface between the surfactant and silica. Due to possible strong electrostatic interactions between the deprotonated curcumin molecules and

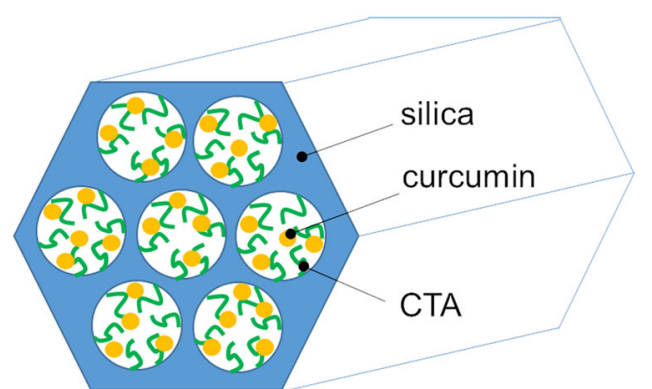

Fig. 8 Schematic representation of the hybrid $\mathrm{Meso}_{-} \mathrm{SiO}_{2}$ Cur material

surfactant cationic heads, curcumin may act as a cotemplating agent in the co-assembly of the mesostructured, so affecting the growth and the morphology of the particles.

\subsection{Release tests}

Since the main aim of the present work is to increase water solubility of curcumin, release tests in different water-based solutions mimicking different physiological conditions were performed.

Even though curcumin is hydrophobic and the curcumin-surfactant interactions are quite strong, the drug could be released from the Meso_SiO ${ }_{2}$ Cur carrier both in PBS and AS (Fig. 7). Interestingly, although curcumin inside the hybrid material is deprotonated, as soon as it is released into an aqueous solution, it becomes protonated, as proved by the UV absorption wavelength of around $420-425 \mathrm{~nm}$ (Fig. 6). Therefore, the hybrid carrier is successful in increasing the solubility of curcumin in water without compromising its efficacy. Indeed, curcumin must be delivered in a protonated form to maintain its beneficial 
properties in the human body since deprotonation is expected to occur during the antioxidant mechanism played by the drug [1].

As far as the release kinetics of the drug, both in PBS and in AS, is concerned, no burst release was observed. The drug release started after a specific lag time ( $1 \mathrm{~h}$ in PBS and $2 \mathrm{~h}$ in AS): it, then, rapidly increased and, finally, reached a plateau. The presence of an initial lag time as well as the absence of residual powder at the end of the test, may induce to think that the release mechanism of curcumin in PBS and in AS involves the degradation of the amorphous silica matrix. On the opposite, almost no curcumin was released in the $\mathrm{HCl}$ solution and a significant amount of yellow powder was retrieved at the end of the test. At acidic $\mathrm{pH}$ the carrier does not undergo any significant degradation process and, therefore, the release amount of curcumin was negligible. Finally, no release at all was observed when crystalline curcumin was dispersed in the solutions; in fact, crystalline curcumin is hydrophobic with very low solubility in water.

As far as the tests in the PBS and AS solutions are concerned, if, as previously hypothesized, curcumin is released upon degradation of the carrier, the active principle is delivered together with silicates and the surfactant micelles. These in solution can confine curcumin and increase its solubility. It is, in fact, reported in the literature that when a critical micellar concentration is overcome, surfactants can solubilize hydrophobic molecules [28, 29]. The presence of curcumin-containing micelles can be proved by the observation of the UV absorption spectrum in AS (Fig. 6). The spectrum, in fact, is comparable to that reported in the literature [6] for curcumin dissolved in CTAmicelles solution.

On the basis of the whole set of results, it can be concluded that the synthesized Meso_SiO ${ }_{2}$ Cur carrier succeeds in protecting and conveying curcumin both in PBS and AS, which mimic plasma and a topical environment, respectively. On the other hand, the carrier results ineffective in releasing the drug in simulated gastric conditions (i.e., a $\mathrm{HCl}$ solution).

Interestingly, once released, curcumin becomes protonated again, recovering its active status. The initial lag time and the absence of powder at the end of the release tests suggest that the release may be also triggered by phenomena of silica degradation. Consequently, as schematically depicted in Fig. 9, not only curcumin, but also silicates and surfactant are released from the hybrid carrier. This can be particularly appealing in view of potential applications of this material in the topical field. Silicon, in fact, can positively affect normal skin formation, since this element displays plastic, trophic, and anti-inflammatory functions [30]. On the other hand, regarding the release of surfactant, it must be pointed out that, at high concentrations, CTA

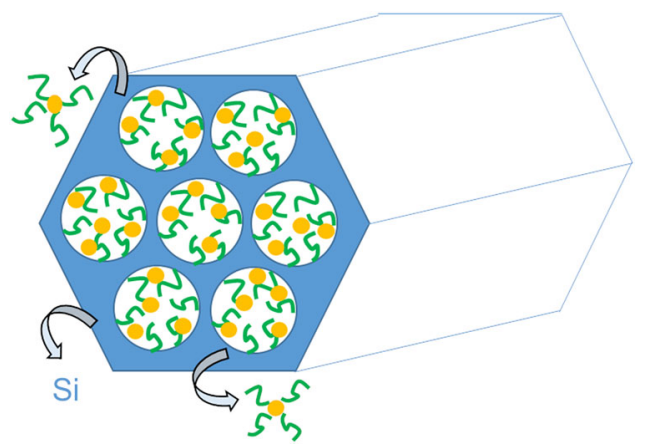

Fig. 9 Schematic representation of the release from hybrid material Meso_SiO 2 _Cur

surfactant can be toxic [31, 32]; therefore, it is envisaged to substitute CTA with a nontoxic surfactant with biological properties. Further studies in this sense are currently under investigation. Nevertheless, the cytotoxicity of CTA could instead be exploited in the formulation of materials for the treatment of cancer or precancer pathologies [33].

\section{Conclusions}

In this study, a hybrid material containing amorphous curcumin, CTA, and silica has been successfully synthesized (Meso_SiO ${ }_{2}$ Cur). In absence of curcumin, a reference hybrid material made of CTA and silica was obtained (Meso_SiO ${ }_{2}$ ). The proposed synthesis process is an organicsolvent-free, rapid one-pot procedure that is conducted at mild conditions and can be easily adapted for the incorporation of other hydrophobic active principles. This novel approach allows the direct incorporation of the active principle during the synthesis process to be obtained, so eliminating the need of any loading procedure and the use of dangerous solvents.

Both the reference material and the curcumin-loaded one are mesostructured. The addition of curcumin results in the modification of the particles morphology. This probably occurs because curcumin acts not as a simple swelling agent, but rather as a co-templating agent, since it assumes a negative charge and can closely interact with the cationic surfactant species.

When $\mathrm{Meso} \_\mathrm{SiO}_{2}$ Cur undergoes the release tests, curcumin is gradually and completely released both in PBS and in AS, where it recovers its protonated active form. Differently from what is usually observed in the literature for silica carriers, the release mechanism of this hybrid material is reasonably governed by a degradation process of the silica matrix. Therefore, not only curcumin, but also surfactant and silicates are released. Thanks to the presence of the surfactant micelles, the water solubility of curcumin is significantly increased. While the release of the cytotoxic 
CTA could be advantageous for the formulation of materials to treat cancer or precancer pathologies, the release of silicates can be beneficial to the skin due to its trophic functions, so confirming the interest of this carrier for topical applications.

Finally, since the carrier does not undergo any significant degradation in the $\mathrm{HCl}$ solution, no curcumin is released in acidic media. This characteristic opens the route for another possible application of the carrier as a gastro protector. Indeed, the proposed carrier could pass through the stomach without releasing the active principle, which could be subsequently released in the intestine where the $\mathrm{pH}$ is less acid.

Acknowledgements The authors would like to thank Mr. Mauro Raimondo for FESEM measurements. Open access funding provided by Politecnico di Torino within the CRUI-CARE Agreement.

Author contributions Conceptualization, MG, FG, and BO; methodology, MG, BO; validation, SR, BO; investigation, MG, FG; resources, LM, BO; data curation, MG, FG; writing-original draft preparation, $\mathrm{MG}$; writing-review and editing, $\mathrm{MB}$ and $\mathrm{BO}$; supervision, $\mathrm{BO}$; funding acquisition, $\mathrm{BO}, \mathrm{MB}, \mathrm{LM}$, and $\mathrm{SR}$.

\section{Compliance with ethical standards}

Conflict of interest The authors declare that they have no conflict of interest.

Publisher's note Springer Nature remains neutral with regard to jurisdictional claims in published maps and institutional affiliations.

Open Access This article is licensed under a Creative Commons Attribution 4.0 International License, which permits use, sharing, adaptation, distribution and reproduction in any medium or format, as long as you give appropriate credit to the original author(s) and the source, provide a link to the Creative Commons license, and indicate if changes were made. The images or other third party material in this article are included in the article's Creative Commons license, unless indicated otherwise in a credit line to the material. If material is not included in the article's Creative Commons license and your intended use is not permitted by statutory regulation or exceeds the permitted use, you will need to obtain permission directly from the copyright holder. To view a copy of this license, visit http://creativecommons. org/licenses/by/4.0/.

\section{Appendix}

Additional figures concerning the synthesis process and washing procedure are here reported.

Figs $10-12$

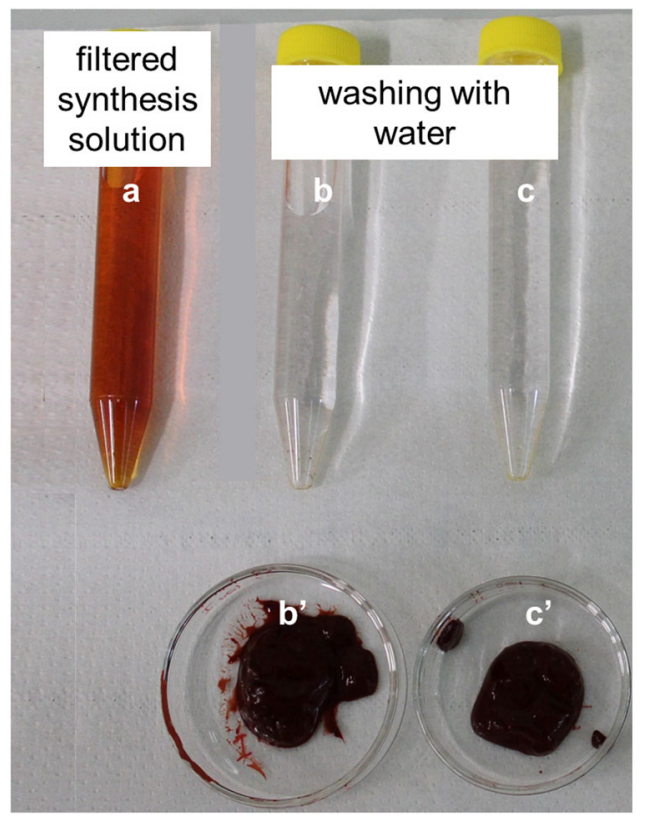

Fig. 10 Filtered synthesis solution of Meso_SiO ${ }_{2}$ Curc (a) and water filtered after washing Meso_SiO2_Curc (once, b, or twice, c) with water. The wet Meso_SiO ${ }_{2}$ Curc material after one $\left(\mathbf{b}^{\prime}\right)$ or two $\left(\mathbf{c}^{\prime}\right)$ washing steps with water are reported in the lower part of the image
Fig. 11 Meso_SiO 2 Curc during (a) and after (b) the washing with ethanol
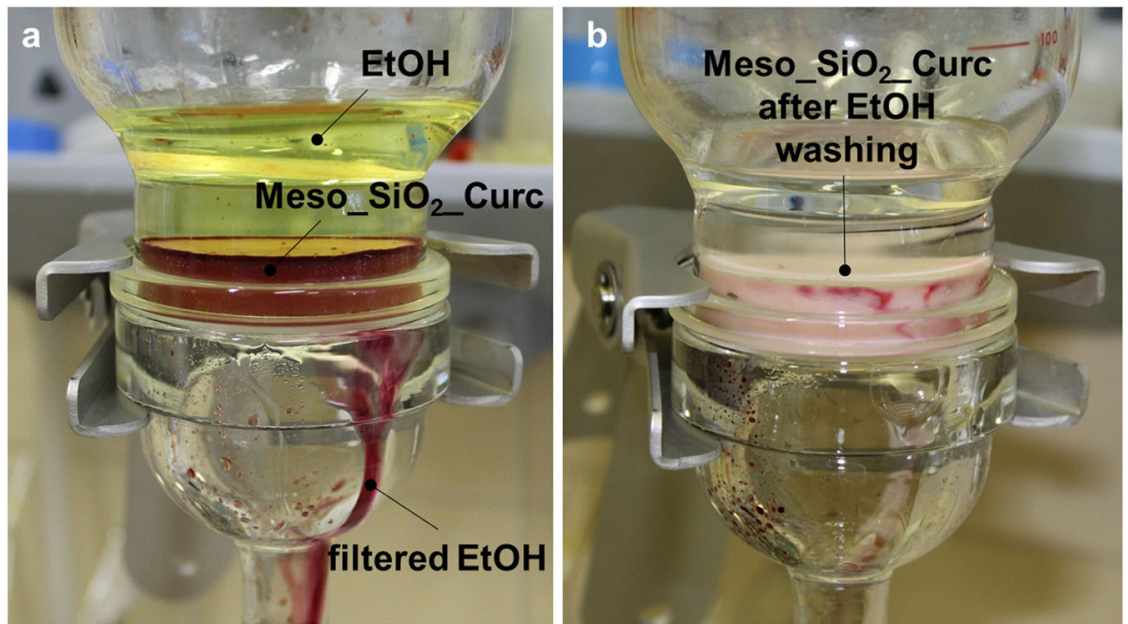


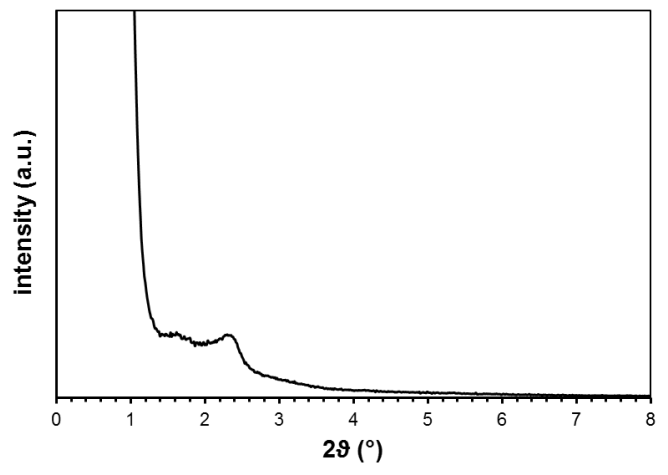

Fig. 12 XRD-pattern of Meso_SiO $\mathrm{Si}_{2}$ Cur at small angles after washing with ethanol

\section{References}

1. Priyadarsini KI (2014) The chemistry of curcumin: from extraction to therapeutic agent. Molecules 19:20091-20112. https://doi. org/10.3390/molecules 191220091

2. Gupta SC, Patchva S, Koh W, Aggarwal BB (2012) Discovery of curcumin, a component of golden spice, and its miraculous biological activities. Clin Exp Pharmacol Physiol 39:283-299. https://doi.org/10.1111/j.1440-1681.2011.05648.x

3. Nguyen TA, Friedman AJ (2013) Curcumin: a novel treatment for skin-related disorders. J Drugs Dermatol 12:1131-1137

4. Sarafian G, Afshar M, Mansouri P, Asgarpanah J, Raoufinejad K, Rajabi M (2015) Topical turmeric microemulgel in the management of plaque psoriasis; a clinical evaluation. Iran J Pharm Res 14:865-876

5. Sun L, Liu Z, Wang L, Cun D, Tong HHY, Yan R, Chen X, Wang R, Zheng Y (2017) Enhanced topical penetration, system exposure and anti-psoriasis activity of two particle-sized, curcumin-loaded PLGA nanoparticles in hydrogel. J Control Release 254:44-54. https://doi.org/10.1016/j.jconrel.2017.03.385

6. Sharma R, Jani D (2013) Interaction of cationic CTAB surfactant with curcumin, an anticarcinogenic drug: spectroscopic investigation. Tenside Surfactants Deterg 50:283-288. https://doi.org/10. 3139/113.110261

7. Singh PK, Wani K, Kaul-Ghanekar R, Prabhune A, Ogale S (2014) From micron to nano-curcumin by sophorolipid co-processing: highly enhanced bioavailability, fluorescence, and anticancer efficacy. RSC Adv 4:60334-60341. https://doi.org/10. 1039/C4RA07300B

8. Leung MHM, Colangelo H, Kee TW (2008) Encapsulation of curcumin in cationic micelles suppresses alkaline hydrolysis. Langmuir 24:5672-5675. https://doi.org/10.1021/la800780w

9. Tiyaboonchai W, Tungpradit W, Plianbangchang P (2007) Formulation and characterization of curcuminoids loaded solid lipid nanoparticles. Int J Pharm 337:299-306. https://doi.org/10.1016/j. ijpharm.2006.12.043

10. Kotcherlakota R, Barui AK, Prashar S, Fajardo M, Briones D, Rodríguez-Diéguez A, Patra CR, Gómez-Ruiz S (2016) Curcumin loaded mesoporous silica: an effective drug delivery system for cancer treatment. Biomater Sci 4:448-459. https://doi.org/10. 1039/C5BM00552C

11. Dhivya R, Ranjani J, Rajendhran J, Mayandi J, Annaraj J (2018) Enhancing the anti-gastric cancer activity of curcumin with biocompatible and $\mathrm{pH}$ sensitive PMMA-AA/ZnO nanoparticles. Mater Sci Eng C 82:182-189. https://doi.org/10.1016/j.msec. 2017.08.058
12. Grynkiewicz G, Ślifirski P (2012) Curcumin and curcuminoids in quest for medicinal status. Acta Biochim Pol 59:201-212. https:// doi.org/10.18388/abp.2012_2139

13. Florek J, Caillard R, Kleitz F (2017) Evaluation of mesoporous silica nanoparticles for oral drug delivery-current status and perspective of MSNs drug carriers. Nanoscale 9:15252-15277. https://doi.org/10.1039/C7NR05762H

14. Jambhrunkar S, Qu Z, Popat A, Yang J, Noonan O, Acauan L, Ahmad Nor Y, Yu C, Karmakar S (2014) Effect of surface functionality of silica nanoparticles on cellular uptake and cytotoxicity. Mol Pharm 11:3642-3655. https://doi.org/10.1021/ mp500385n

15. Zhan S, Li S, Zhao Q, Wang W, Wang J (2017) Measurement and correlation of curcumin solubility in supercritical carbon dioxide. J Chem Eng Data 62:1257-1263. https://doi.org/10.1021/acs.jced. 6b00798

16. Gangwar RK, Tomar GB, Dhumale VA, Zinjarde S, Sharma RB, Datar S (2013) Curcumin conjugated silica nanoparticles for improving bioavailability and its anticancer applications. J Agric Food Chem 61:9632-9637. https://doi.org/10.1021/jf402894x

17. Kerkhofs S, Saïdi F, Vandervoort N, Van den Mooter G, Martineau C, Taulelle F, Martens JA (2015) Silica capsules enclosing P123 triblock copolymer micelles for flurbiprofen storage and release. J Mater Chem B 3:3054-3061. https://doi.org/10.1039/ C5TB00058K

18. Chin SF, Iyer KS, Saunders M, St. Pierre TG, Buckley C, Paskevicius M, Raston CL (2009) Encapsulation and sustained release of curcumin using superparamagnetic silica reservoirs. Chem Eur J 15:5661-5665. https://doi.org/10.1002/chem. 200802747

19. National Toxicology Program U.S (2008) NTP Technical Report on the Toxicology and Carcinogenesis Studies of Formamide (CAS No. 75-12-7) in F344/N Rats and B6C3F1 Mice (Gacave Studies). https://ntp.niehs.nih.gov/ntp/htdocs/lt_rpts/tr541.pdf

20. Onida B, Bonelli B, Flora L, Geobaldo F, Arean CO, Garrone E (2001) Permeability of micelles in surfactant-containing MCM41 silica as monitored by embedded dye molecules. Chem Commun 2216-2217. https://doi.org/10.1039/B105261F

21. Cai Q, Lin W-Y, Xiao F-S, Pang W-Q, Chen X-H, Zou B-S (1999) The preparation of highly ordered MCM-41 with extremely low surfactant concentration. Microporous Mesoporous Mater 32:1-15. https://doi.org/10.1016/S1387-1811(99)00082-7

22. Shimamura $T$, Tairabune $T$, Kogo $T$, Ueda $H$, Numajiri $S$, Kobayashi D, Morimoto Y (2004) Investigation of the release test method for the topical application of pharmaceutical preparations: release test of cataplasm including nonsteroidal anti-inflammatory drugs using artificial sweat. Chem Pharm Bull 52:167-171. https://doi.org/10.1248/cpb.52.167

23. Cai Q, Luo Z-S, Pang W-Q, Fan Y-W, Chen X-H, Cui F-Z (2001) Dilute solution routes to various controllable morphologies of MCM-41 silica with a basic medium. Chem Mater 13:258-263. https://doi.org/10.1021/cm990661z

24. Viana R, Silva A, Pimentel A (2012) Infrared spectroscopy of anionic, cationic, and zwitterionic surfactants. Adv Phys Chem 2012:903272. https://doi.org/10.1155/2012/903272

25. Mangolim CS, Moriwaki C, Nogueira AC, Sato F, Baesso ML, Neto AM, Matioli G (2014) Curcumin- $\beta$-cyclodextrin inclusion complex: stability, solubility, characterisation by FT-IR, FTRaman, X-ray diffraction and photoacoustic spectroscopy, and food application. Food Chem 153:361-370. https://doi.org/10. 1016/j.foodchem.2013.12.067

26. Dhakal S, Schmidt FW, Kim M, Tang X, Peng Y, Chao K (2019) Detection of additives and chemical contaminants in turmeric powder using FT-IR spectroscopy. Foods 8. https://doi.org/10. 3390/foods 8050143 
27. Sokolov I, Kievsky YY, Kaszpurenko JM (2007) Self-assembly of ultrabright fluorescent silica particles. Small 3:419-423. https:// doi.org/10.1002/smll.200600319

28. Wang Z, Leung M, Kee T, English D (2009) The role of charge in the surfactant-assisted stabilization of the natural product curcumin. Langmuir 26:5520-5526. https://doi.org/10.1021/la 903772e

29. Rahman SMH, Telny TC, Ravi TK, Kuppusamy S (2009) Role of surfactant and $\mathrm{pH}$ in Dissolution of Curcumin. Indian J Pharm Sci 71:139-142. https://doi.org/10.4103/0250-474X.54280

30. Seaborn CD, Nielsen FH (2002) Silicon deprivation decreases collagen formation in wounds and bone, and ornithine transaminase enzyme activity in liver. Biol Trace Elem Res 89:251-261
31. Yildirim A, Turkaydin M, Garipcan B, Bayindir M (2016) Cytotoxicity of multifunctional surfactant containing capped mesoporous silica nanoparticles. RSC Adv 6:32060-32069. https://doi.org/10.1039/C5RA21722A

32. Jia YP, Shi K, Liao JF, Peng JR, Hao Y, Qu Y, Chen LJ, Liu L, Yuan X, Qian ZY et al. (2020) Effects of cetyltrimethylammonium bromide on the toxicity of gold nanorods both in vitro and in vivo: molecular origin of cytotoxicity and inflammation. Small Methods 4:1900799. https://doi.org/10.1002/ smtd.201900799

33. He Q, Shi J, Chen F, Zhu M, Zhang L (2010) An anticancer drug delivery system based on surfactant-templated mesoporous silica nanoparticles. Biomaterials 31:3335-3346. https://doi.org/10. 1016/j.biomaterials.2010.01.015 